\title{
THE MECHANISMS PRODUCING FRACTURE-SEPARATION OF THE PROXIMAL HUMERAL EPIPHYSIS
}

\author{
DAVID J. WILLIAMS \\ From Northwick Park Hospital and Clinical Research Centre, Harrow
}

\begin{abstract}
Six mechanisms which may produce fracture-separation of the proximal humeral epiphysis are proposed. Four of them are illustrated by case reports. It is suggested that they may be differentiated by examination of the patient and study of the radiographs. In unstable fractures manipulation is indicated and, if performed with an understanding of the mechanism of injury, will produce a stable reduction.
\end{abstract}

Fracture-separation of the upper humeral epiphysis is uncommon (Neer and Horwitz 1965). The injury can occur at any age while the proximal epiphysis remains open, but occurs with greatest frequency in boys between the ages of 11 and 17 years. The difference in the incidence between the sexes is probably related to the aetiological factors - contact sports, such as football and wrestling, and falls from heights (Smith 1956).

The mechanisms which produce the injury are not clear but can be classified as adduction or abduction types (Stewart and Hundley 1955; Crenshaw 1971; Wilson 1976); however, attempts to reduce these fractures by applying an opposite force have failed. Neer and Horwitz (1965) considered that the mechanism was a direct shearing force on the shoulder, while Bourdillon (1950) and Aitken (1963) both believed that lateral rotation and extension of the arm were the deforming forces.
The purpose of this report is to define the possible mechanisms of injury which cause fracture-separation of the upper humeral epiphysis and to illustrate these with case reports.

\section{MECHANISMS ILLUSTRATED BY CASE REPORTS}

\section{Forced lateral rotation and extension}

Case 1. A 10-year-old boy fell backwards from a height of four feet, onto his outstretched right arm. Examination showed the arm to be abducted and extended with anteromedial bruising and a swelling of the shoulder which was found to be the proximal end of the metaphysis. Radiographs (Figs 1 and 2) confirmed a fractureseparation of the proximal humeral epiphysis with the metaphysis lying anteromedially. The fracture was reduced with the aid of the image intensifier, by hyperextension, traction, flexion and medial rotation. The arm was immobilised in medial rotation and flexion, first with a collar and cuff and Elastoplast strapping for one week and then as shown in Figure 3. At three weeks the patient could abduct the arm

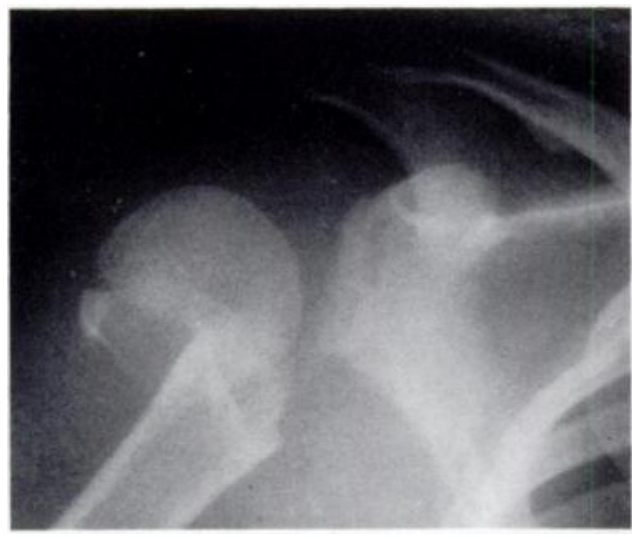

Fig. 1

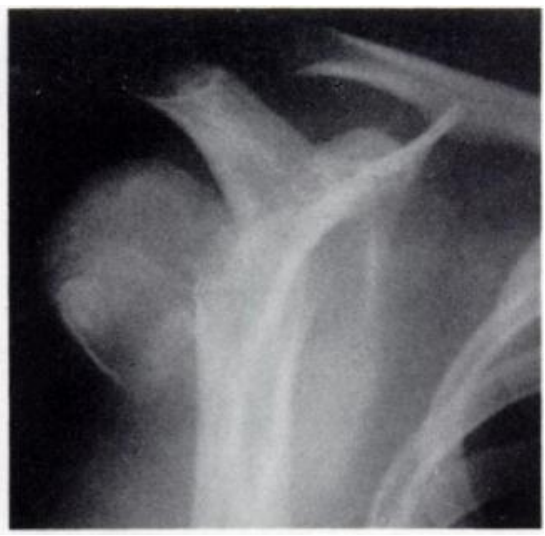

Fig. 2

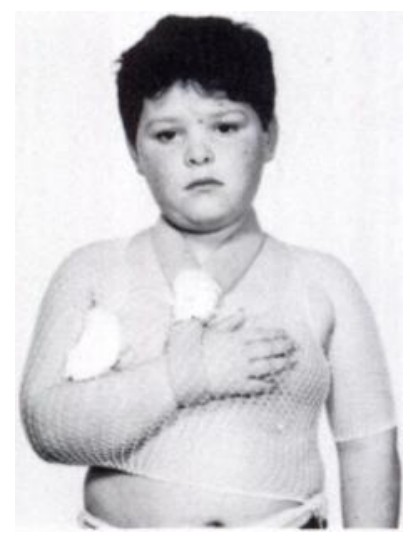

Fig. 3

Case 1. Injury in lateral rotation and extension. Figure 1-Anteroposterior radiograph of the injury showing the upper metaphysis lying medially and the triangular fragment of intact metaphysis with an attached periosteum lying laterally. Figure 2-Lateral view of the scapula showing the upper metaphysis lying anteriorly and the triangular fragment attached to the epiphysis lying posteriorly. Figure $3-$ The position of immobilisation.

D. J. Williams, FRCS, Senior Orthopaedic Registrar, Northwick Park Hospital and Clinical Research Centre, Watford Road, Harrow, Middlesex, HAI 3 UJ, England.

(C) 1981 British Editorial Society of Bone and Joint Surgery 0301-620X/81/00230102\$2.00 


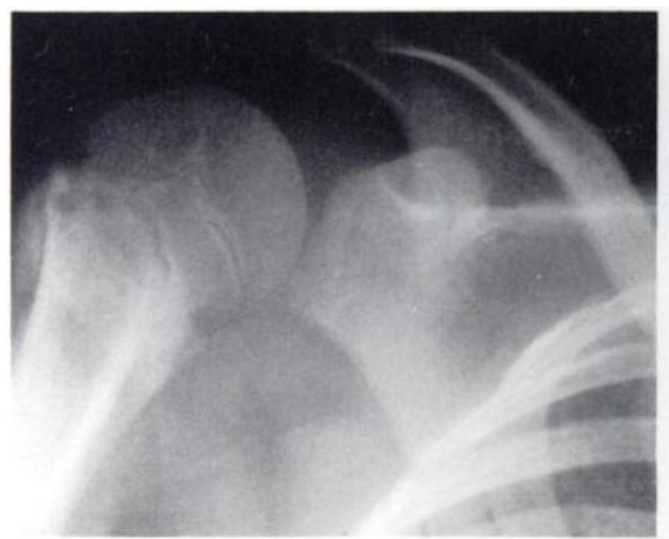

Fig. 4

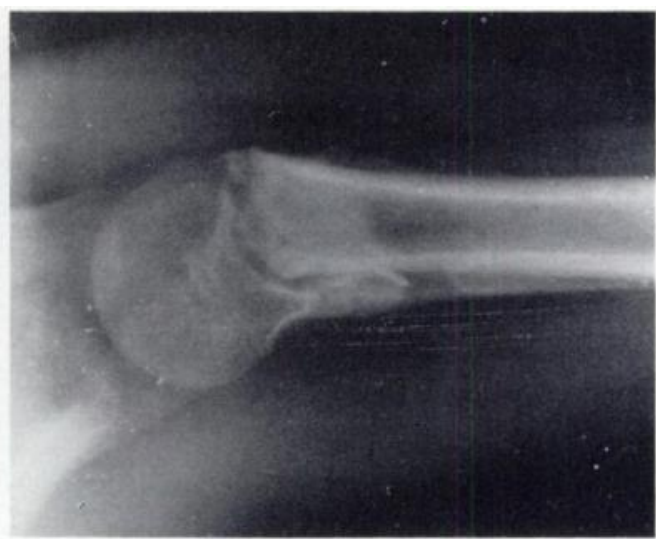

Fig. 5

Case 1. Figure 4-At seven weeks the anteroposterior radiograph shows marked periosteal new bone. Figure 5 - The axial radiograph shows the final anterior angulation at the fracture site.

to 90 degrees but radiographs showed some anterior angulation of the fracture. The arm was left free, and after seven weeks there was a full range of movement and the angulation had not increased (Figs 4 and 5).

\section{Forced medial rotation and flexion}

Case 2. A nine-year-old boy was thrown over the handlebars of his bicycle, holding his left elbow close to his side. There was posterolateral bruising with a swelling which was found to be the upper end of the metaphysis. Radiographs (Figs 6 and 7) confirmed a fracture-separation with the metaphysis lying posterolaterally. This was reduced by traction, lateral rotation and extension. This position was held by an abduction splint (Fig. 8) for three weeks; the patient was then readmitted to bring the arm to his side. After $\mathbf{4 8}$ hours he was discharged home in a collar and cuff with instructions to start mobilising the shoulder. At six weeks he had a full range of movement of the shoulder and the radiographs showed no displacement (Figs 9 and 10$)$.

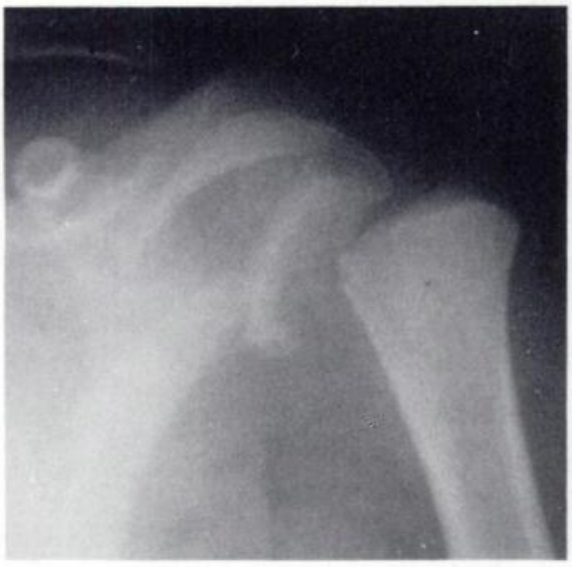

Fig. 6

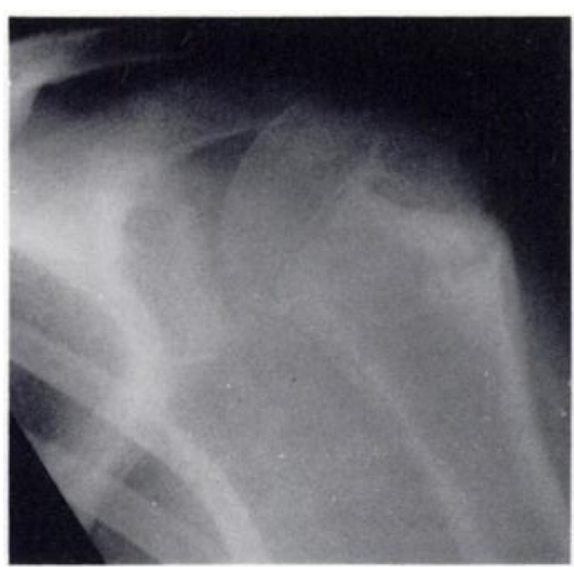

Fig. 9

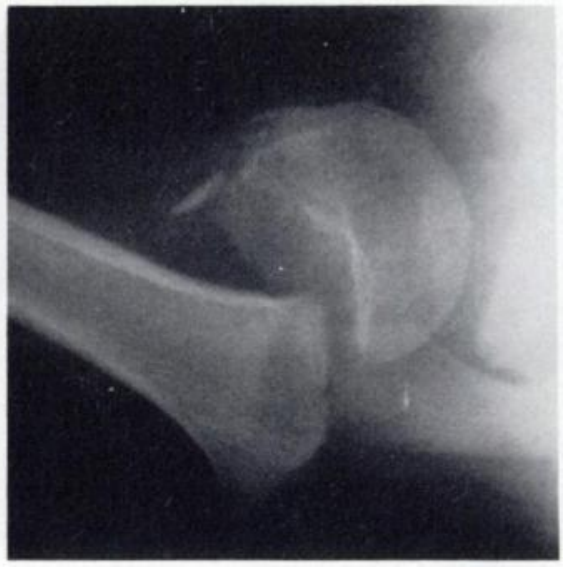

Fig. 7

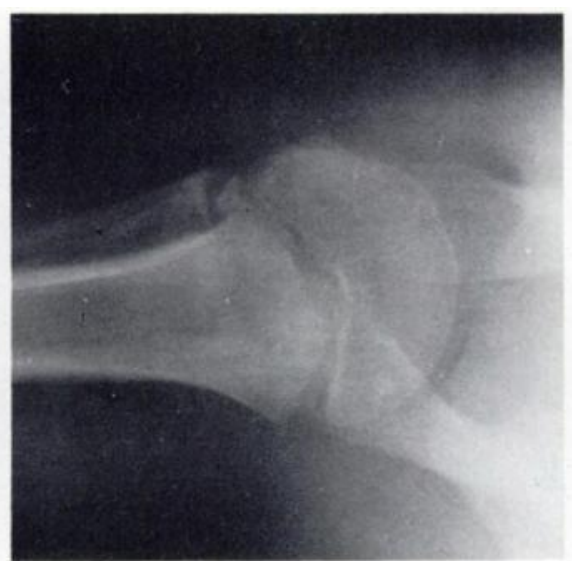

Fig. 10

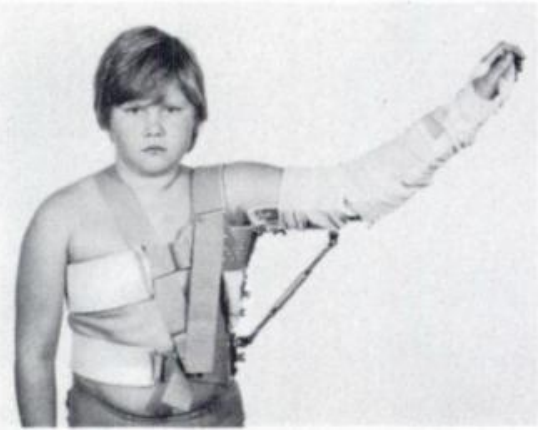

Fig. 8

Case 2. Injury in medial rotation and flexion.

Figure 6-Anteroposterior radiograph of the injury showing the upper metaphysis lying laterally and the triangular fragment of intact metaphysis with attached periosteum lying medially. Figure $7-$ The axial radiograph showing the upper metaphysis displaced posteriorly and the triangular fragment lying anteriorly. Figure 8-The position of appliance used to maintain reduction. Figures 9 and 10-Anteroposterior and axial radiographs at six weeks. 


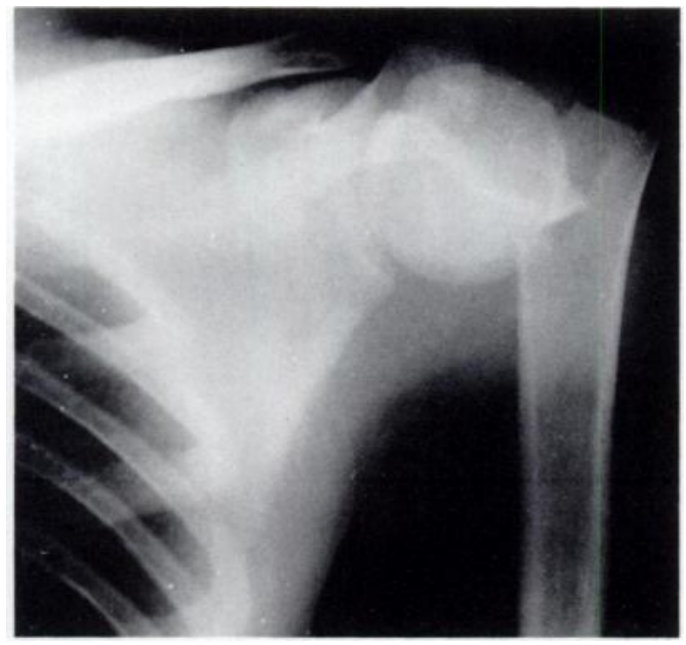

Fig. 11

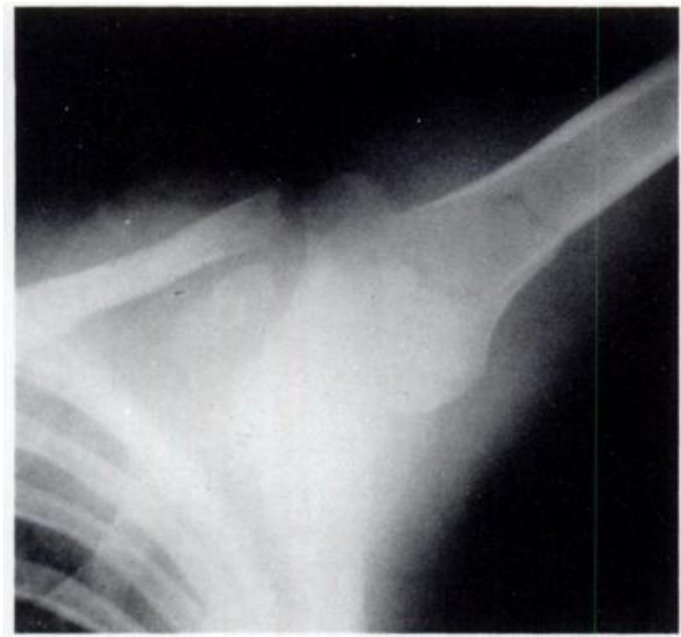

Fig. 13

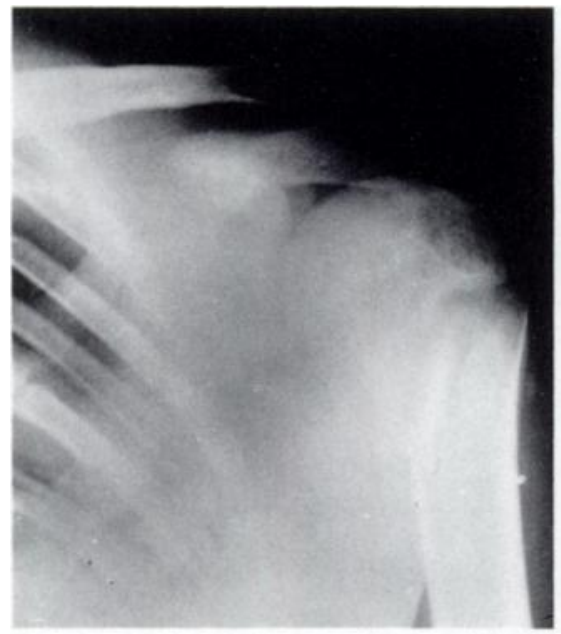

Fig. 15

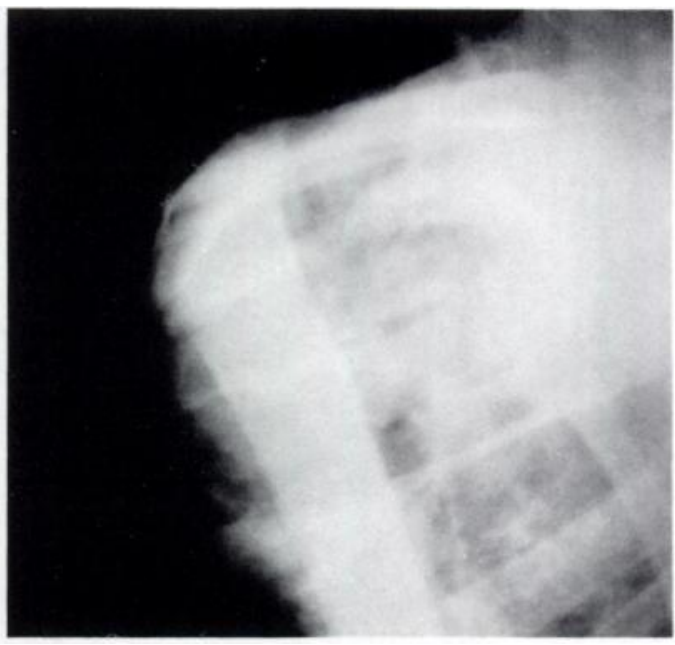

Fig. 12

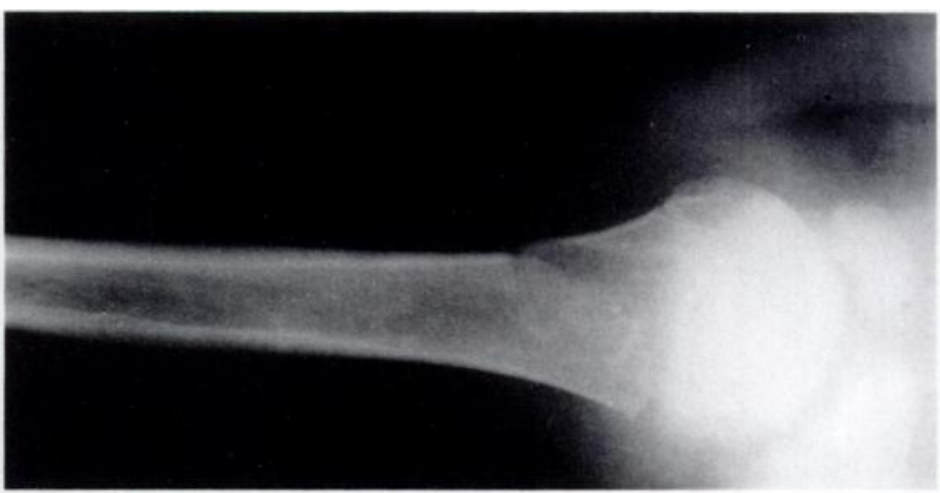

Fig. 14
Case 3.

Injury in

medial rotation with extension.

Figure 11-Anteroposterior radiograph showing the upper metaphysis lying laterally with the triangular fragment of metaphysis lying medially. Figure 12-Shoot-through lateral radiograph showing anterior displacement of the metaphysis with the triangular fragment and attached metaphysis lying posteriorly. Figures 13 and 14-Anteroposterior and axial radiographs showing the fracture reduced. Figure 15-Some loss of reduction. Figure 16-Some recurring anterior angulation. 


\section{Forced medial rotation with extension}

Case 3. This 17-year-old boy fell 15 feet backwards through a roof, landing with his left elbow flexed and behind him. There was marked bruising and swelling of the anterior lateral aspect of the shoulder which, when palpated, was found to be the upper metaphysis penetrating the deltoid. Radiographs (Figs 11 and 12) confirmed a fracture-separation with the metaphysis lying anterolaterally. Manipulation by traction, lateral rotation and flexion reduced the fracture (Figs 13 and 14). A plaster-of-Paris cast held this position but subsequently he developed a palsy of the ulnar nerve. The cast was therefore replaced by a collar and cuff with some subsequent loss of reduction of the fracture (Figs 15 and 16). Full recovery of the neurological deficit occurred. Active lateral rotation was encouraged after two weeks, and by seven weeks there was minimal loss of range of lateral rotation.

\section{Pure extension}

Case 4. A 12-year-old boy fell four feet and, as he did so, his left elbow struck a stool which forced his arm into extension. Examination showed that there was bruising of the arm and an anterior swelling which, when palpated, was found to be the upper end of the metaphysis. Radiographs (Figs 17 and 18) confirmed the fractureseparation with the metaphysis showing no medial or lateral displacement, but there was anterior angulation at the fracture site. As the fracture was clinically stable, no attempt at reduction was made and the patient was treated in a collar and cuff. No subsequent change

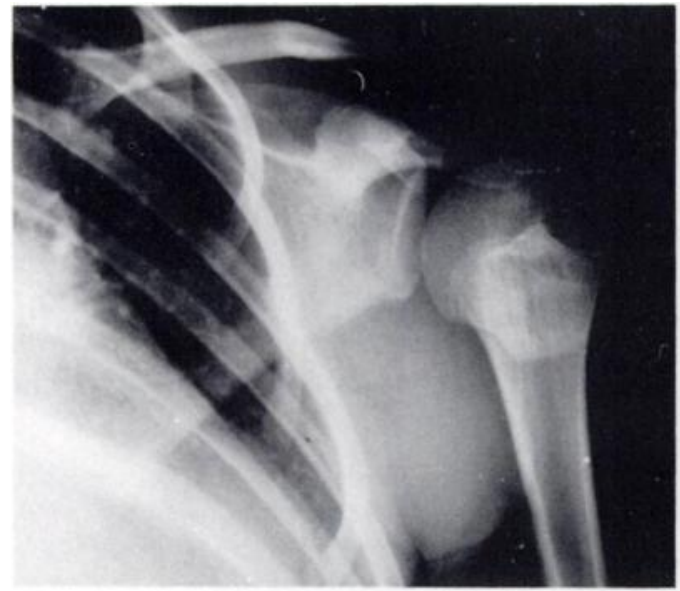

Fig. 17

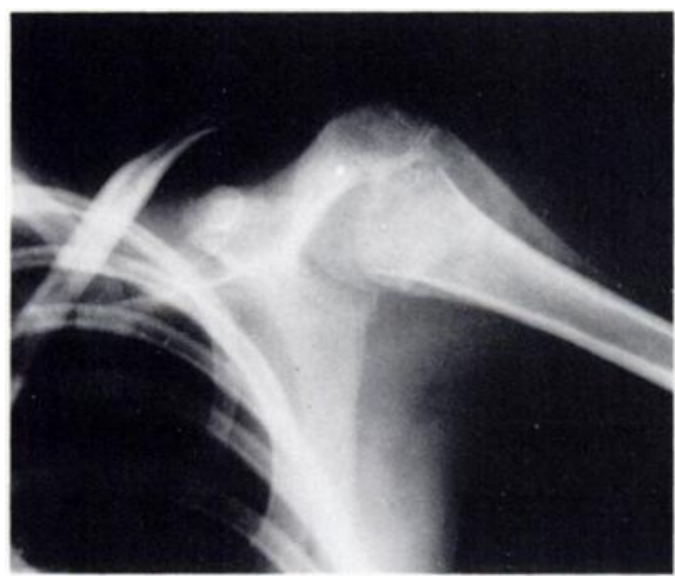

Fig. 19 in the deformity occurred while the fracture was uniting. After three weeks, the shoulder was mobilised, but the deformity persisted (Figs 19 and 20). Functionally, the arm had a satisfactory range of movement, but there was still about 30 degrees loss of flexion. It is expected that some of this will improve with remodelling.

\section{DISCUSSION}

Standard reference books (Crenshaw 1971; Wilson 1976) continue to classify the fractures of the proximal humeral epiphysis as abduction or adduction types, but it is anatomically impossible to abduct the arm beyond 90 degrees without lateral rotation or to adduct beyond neutral without rotating the arm into lateral or medial rotation.

Neer and Horwitz (1965) considered that a direct shearing force was the mechanism of injury, on the basis of the case histories of two-thirds of their patients. However, Neer and Rockwood (1975) later stated that the patient was often unable to recall the details of the accident and it was difficult to deduce the mechanism from an incomplete history. Further, direct injury to the upper arm usually causes a transverse fracture of the

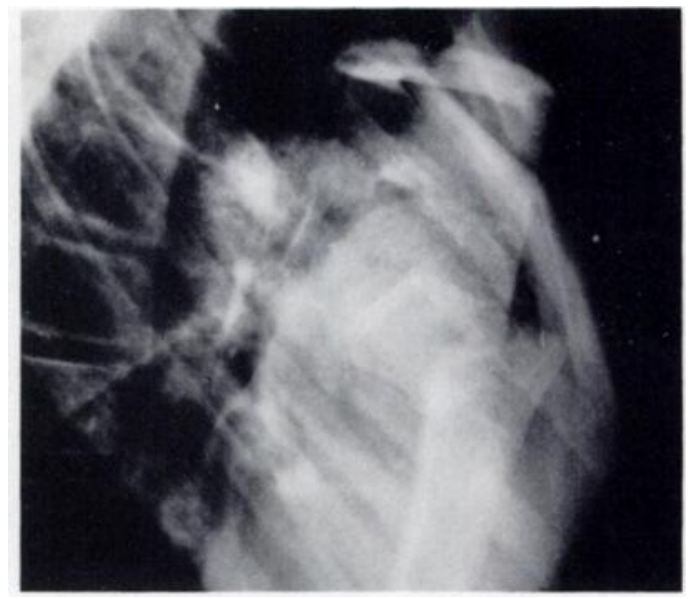

Fig. 18

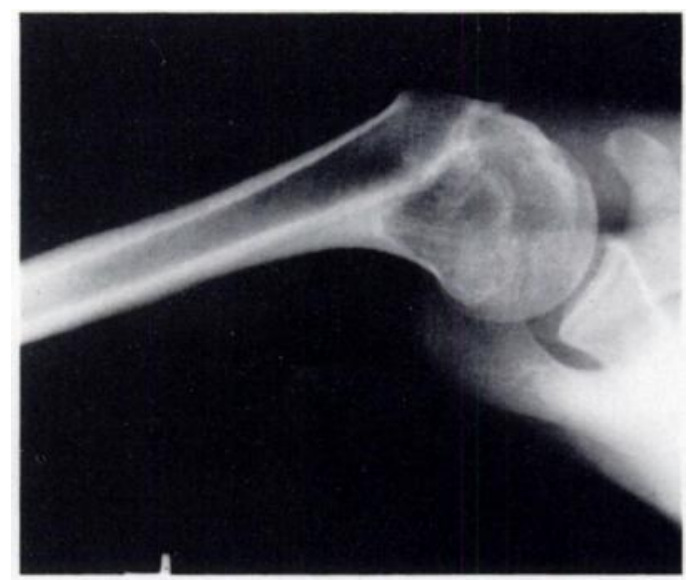

Fig. 20

Case 4. Extension injury. Figure 17-Anteroposterior radiograph showing no medial or lateral displacement of metaphysis but loss of the normal epiphysial line. Figure 18-Shoot-through lateral radiograph showing marked anterior angulation of the fracture with, posteriorly, the undisplaced metaphysial fragment. Figures 19 and $20-$ The healed position at six weeks, with persisting deformity. 


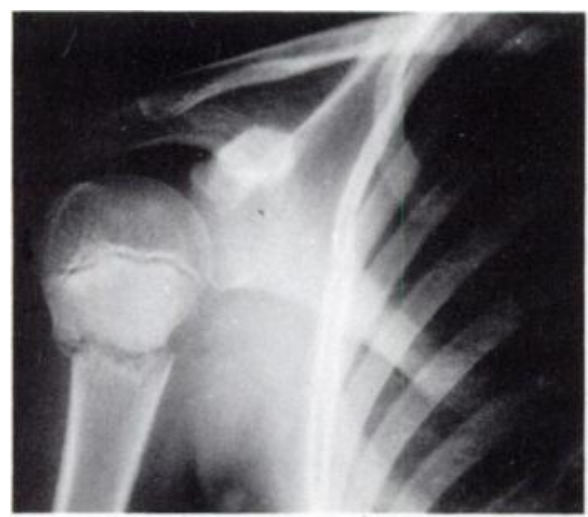

Fig. 21

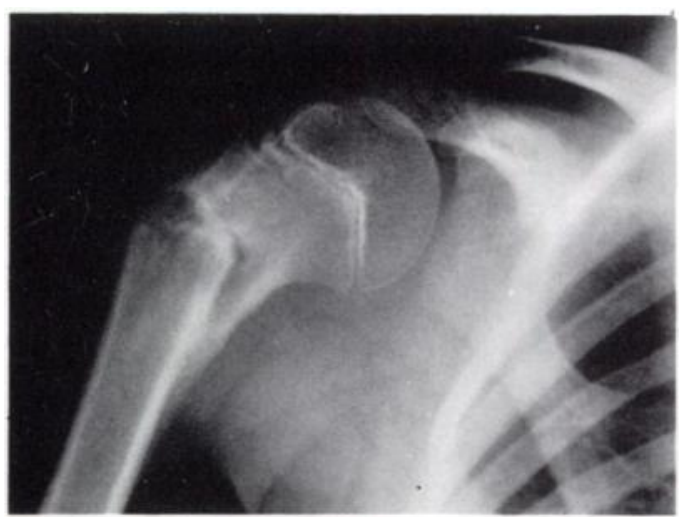

Fig. 22

Figure $21-A$ transverse fracture of the upper shaft due to a direct shearing force. Figure $22-$ Healing of this fracture.

humerus close to, but not involving, the epiphysis (Figs 21 and 22); this is well recognised for all other long bones. Tachdjian (1972) supports the view that indirect violence is the mechanism of injury.

Bourdillon (1950) was the first to discuss the mechanism of injury and, using the evidence of case reports, suggested that extension and lateral rotation were the deforming forces. Aitken (1963) supported this view but experienced difficulty in reducing some fractures, which may be explained by his failure to appreciate that there might be other mechanisms.

I believe that the injury is produced by a combination of one or more of four forces: forced extension, forced flexion, forced lateral rotation, and forced medial rotation. The first pair are mutually exclusive, as are the second pair. There are therefore eight possible combinations: pure extension, pure flexion, pure forced lateral rotation, pure forced medial rotation, forced extension with lateral rotation, forced extension with medial rotation, forced flexion with lateral rotation, and forced flexion with medial rotation. It seems unlikely that pure forced lateral rotation or pure forced medial rotation alone will occur in practice. Probably only physical assault will produce either of these as an isolated force. There are therefore six mechanisms of injury, four of which have been illustrated by the case reports.

Radiographs taken in two planes at 90 degrees to each other are essential to deduce the mechanism of injury. Bourdillon (1950), Smith (1956) and Campbell and Almond (1977) all emphasised that radiographs taken in one view only could be misleading. Neer (1970) reported a method of obtaining the necessary radiographs without moving the arm of the patient, who may be placed standing, sitting or lying down.

Management. Smith (1956) was the first to advocate the laissez faire approach to the management of this injury. He based his argument on the difficulty of obtaining and maintaining reduction of the fracture; in his series 50 per cent of the fractures on which open reduction was performed subsequently redisplaced but the extensive remodelling that took place corrected much of the deformity. The shortening that resulted was accepted. Neer and Horwitz (1965), from the same hospital, suggested that manipulation should be carried out for those fractures that the first radiograph showed to be greatly displaced. Nilsson and Svartholm (1965) also held this view, while Tachdjian (1972) suggested that angulation of more than 20 degrees should be corrected.

It has been my experience that these fractures may be clinically divided into two groups: stable and unstable fractures. Stable fractures are those which cause least discomfort; these may produce obvious deformity, but the child allows the examiner to abduct the shoulder passively to 90 degrees. This type of fracture does not require manipulation (Case 4 ). The child with the unstable fracture appears in great pain and will not allow passive abduction to 90 degrees. In this group, the degree of displacement is invariably complete and requires manipulation. The principle of manipulation is to reverse the mechanism of injury, thus automatically producing a stable reduction.

In conclusion, six mechanisms of fractureseparation of the upper humeral epiphysis are proposed. These may be recognised by examination of the patient and study of suitable radiographs. Unstable fractures, which have the greatest displacement and cause severe discomfort for the patient, should be manipulated and immobilised in the position of stability, which may be deduced from the mechanism of injury. Failure to understand the mechanism must lead to failure of manipulation. 


\section{REFERENCES}

Aitken AP. Fractures of the proximal humeral epiphysis. Surg Clin North Am 1963;43:1573-80.

Bourdillon JP. Fracture-separation of the proximal epiphysis of the humerus. J Bone Joint Surg [Br] 1950;32-B:35-7.

Campbell J, Almond HGA. Fracture-separation of the proximal humeral epiphysis. J Bone Joint Surg [Am] 1977;59-A:262-3.

Crenshaw AH, ed. Campbell's operative orthopaedics. 5th ed. Vol. 1. St Louis: CV Mosby Co, 1971.

Neer CS II. Displaced proximal humeral fractures. I. Classification and evaluation. J Bone Joint Surg [Am] 1970;52-A:1077-89.

Neer CS II, Horwitz BS. Fractures of the proximal humeral epiphysial plate. Clin Orthop 1965;41:24-31.

Neer CS II, Rockwood CA Jr. Fractures of the proximal humerus. In: Rockwood CA Jr, Green DP, eds. Fractures. Philadelphia: Lippincott \& Co, 1975;1:585-610.

Nilsson S, Svartholm F. Fractures of the upper end of the humerus in children. Acta Chir Scand 1965;130:433-9.

Stewart MJ, Hundley JM. Fractures of the humerus: a comparative study in methods of treatment.J Bone Joint Surg [Am] 1955;37-A:681-92.

Smith FM. Fracture-separation of the proximal humeral epiphysis. Am J Surg 1956;91:627-35.

Tachdjian MO. Paediatric orthopaedics. Philadelphia: WB Saunders Co Ltd, 1972;II:1555-60.

Wilson JN, ed. Watson-Jones. Fractures and joint injuries. 5th ed. Edinburgh, London and New York: Churchill Livingstone, 1976 ;II:548. 